\title{
Radiocarbono y Calibración: Potencialidades para la arqueología andina
}

\section{Elmo León Canales*}

\section{Resumen}

Este artículo se basa en gran parte en el manual maestro de Taylor (1987) sobre radiocarbono, aun vigente a pesar del tiempo. No pretende, por tanto, ser exhaustivo en bibliografia, sino simplemente citar algunos textos básicos a modo de referencia. La segunda parte, menor y complementaria a la primera, es un ejercicio de calibración sólo sobre un episodio de la arqueología andina, en función de sopesar la importancia de examinar eventos pasados en años calendarios, en lugar de radiocarbónicos. La finalidad última de este ensayo es la de incentivar a los graduandos para aplicar calibración no sólo en sus investigaciones, sino también en sus tesis y trabajos monográficos, a fin de empezar a evaluar nuestra historia a partir de años siderales reales, y no sólo radiocarbónicos, que es lo que suele ser más común.

Palabras claves: Radiocarbono, calibración radiocarbónica, arqueología andina.

\begin{abstract}
This paper is mainly based on the handbook written by Dr. Taylor (1987) on radiocarbon, which is still in force despite of the years. It does not covers all the literature, but quotes some basic texts as references. The second part of the text, which is shorter and complementary to the first, is an exercise of calibration on a an eposide of andean archaeology, as a means to evaluate the importanc of examining past events on calendar years instead of radiocarbon ones. The final aim of this essay is to motivate students to apply calibration in their research, as well as in their thesis and monographic works, in order to start evaluating our history on the basis of astral years and not only radiocarbon ones, which is currently the usual approach.
\end{abstract}

Keywords: Radiocarbon, radiocarbon calibration, andean archaeology.

*Elmo León Canales. Jr. Los Mogaburos 247. Dpt. 504. Lima 11. Perú. Correoelectrónico: elmoleon@gmx.net 


\section{Introducción}

Hace 4 años se conmemoró el 50 aniversario de la publicación y la consiguiente divulgación del método del radiocarbono por medio de la publicación del libro Radiocarbon Dating (Libby 1952). La primera muestra analizada (C-1) fue un trozo de madera de acacia de la tumba del faraón Zoser (3ra. dinastía) de Sakkara, Egipto, dando un resultado de 4650 \pm 75 BP (Braiwood et al 1951: 53). A pesar de la mínima diferencia con respecto del fechado histórico, el método probó su eficacia, que fue recibida por la comunidad con entusiasmo (Collier 1952, Heizer 1956).

Algunos años antes (1949), Arnold y Libby habían dado a conocer los primeros resultados radiocarbónicos en su famoso artículo "Age Determinations by Radiocarbon Content: Checks with Samples of Known Age" publicado en la revista Science. Estas primeras pruebas comparaban fechados radiocarbónicos con históricos, sugiriendo la validez del radiocarbono como método de fechado.

Es desde ese primer momento hasta nuestros días - con avances como la recalibración de la secuencia de Egipto Pre-Dinástico (Savage 2001) o la recalibración del inicio de sociedades agro-pastoriles en Cercano Oriente hacia el $8,000 \mathrm{cal} A C$, con el despegue de la revolución urbana en Mesopotamia hacia el 6,000 cal AC (Aurenche et al 2001), hasta el descubrimiento que los primeros humanos se dispersaron más rápido desde Eurasia hacia Europa, en un lapso de $\tan$ solo 4,000 años calendarios en el Pleistoceno (Mellars 2006) - que el radiocarbono no ha cesado de brindar sus grandes aportes a prehistoriadores y arqueólogos.

El tremendo impulso cobrado por el radio. carbono generó una serie de proyectos para fechar muestras de distintos contextos, en un inicio del Mediterráneo, y probar la eficacia del radiocarbono. No tardó mucho en descubrirse lo que algunos han llamado la "segunda revolución radiocarbónica", i.e. la demostración por dendrocronología de que la producción de $\mathrm{C}^{14}$ bios- férico no había sido constante ni temporal ni espacial, por lo que era necesario corregir o calibrar los fechados $C^{\text {Ht }}$ (De V ries 19533), tanto para la arqueología internacional, como la del área andina.

Tal vez el mismo Libby nunca imaginó las repercusiones de semejante descubrimiento no sólo en el campo de la arqueología, sino también en el de la biología. La posibilidad de fechar a especímenes orgánicos con el fin de saber cuando fue el momento de su muerte, abrió un gran campo en las ciencias prehistóricas (Currie 2004). Más aún, la adición de la calibración radiocarbónica convierte al método en una herramienta capaz de ubicar en el tiempo, a escala de precisión anual, diferentes eventos desde el Pleistoceno hasta la actualidad, tanto en el Hemisferio Norte (Reimer et al 20C14) como también en el Sur, incluidos los Andes (MacCormac et al 2002, 2004).

Por consiguiente, la nueva curva de calibración nos coloca por primera vez en condición de determinar con mucha más precisión eventos del pasado precolombino para evaluar sus verdaderas cronologías y descubrir sus reales conexiones y diferencias temporales. Son las calibracinnes radiocarbónicas las que nos harán posible responder a la pregunta elementall en arqueologia: icuándo sucedió esto?

Radiocarbono: La "bomba atómica arqueológica"

Así fue calificado el método radiocarbónico por Johnsun a finales de la década de 1940 (Johnson 1965:762). Según Taylor, si hay una fecha a celebrar como el aniversario del método es el 12 de julio de 1948, cuando una muestra egipcia fue la primera en fecharse por el radiocarbono (Taylor 2000: 2). Su descubrimiento solo podría ser equiparado al de la gran antigüedad de la especiehumana (Daniel 1967: 266).

Lia historia se remonta a mediados de la década de 1930, cuando Korff examina la naturaleza y radioactividad de los rayos cósmicos (Taylor 1987: 151). Resulta curioso en este contexto, 
que un episodio bélico como la Segunda Guerra Mundial promoviera investigaciones nucleares, que terminarían por beneficiar el conocimiento del radiocarbono. Es un buen ejemplo de cómo coyunturas negativas pueden aportar significativamante a la ciencia.

Parte de esta cadena de eventos fortuitos es la nominación de Libby como profesor de química en el Departamento de Química del Instituto de Estudios Nucleares en la Universidad de Chicago. No obstante, según Taylor, el proyecto $\mathrm{C}^{\text {if }}$ fue en un principio bastante discreto. Prohablemente el primer reporte relacionado con ello, y donde él resume todo su propósito, fue en un trabajo publicado en junio de 1946 en Physical Review. Aquí, Libby no sólo postula la vida media radiocarbónica, sino también la diferencia de con tenido $\mathrm{C}^{1 ;}$ entre organismos vivientes y carbón fósil, el núcleo de la teoría radiocarbónica (Libby 1946).

Aparentemente, la primera vez que Libby comentó a sus colaboradores sobre su intención de fechar restos orgánicos por medio del radiocarbono fue a fines de 1946. Fue entonces que James Arnold, colaborador de Libby, se lo mencionó al director del Museo Metropolitano de Nueva York, Ambrose Lansing, quien de inmediato le solicitó el fechado de algunas muestras. El request de Lansing fue la primera solicitud de esta índole dirigida a Libby en Chicago, en enero de 1947. El paquete contenía lo que seria la primera muestra para fechar con el código C-1: un trozo de madera de ciprés de la tumba de Sakkara del fararón Zoser de la Tercera Dinastía Egipcia.

Resulta de interés para el área andina, que uno de sus principales investigadores, Paul Fejos, fuera quien posibilitara el desarrollo del programa de Libby' en sus inicios. La necesidad de fondos para continuar con el proyecto puso a Libby, por mediode su mentor, Harold Urey, en contacto con Fejos, por ese entonces director del Viking Found for Anthropological Research. A fines de 1947. Libby, Anderson y Arnold se hallaban en plena investigación, cuando Fejos invita a Libby a dar un lecture en Nueva York para el año siguiente. Taylor cuenta que luego de presentar el método, el público enmudeció y sólo el arqueólogo Richard Foster se vio sumamente interesado y hasta solicitó a Libby el envio de muestras. Arnold y Libby se dieron luego cuenta de que probablemente eran las grandes cantidades de carbón que se necesitaba para fechar, lo que incomodó a los arqueólogos en el auditorio, mas aún a los: "...perros custodios de los Museos que no darian muestras a fechar" (Taylor 1987: 155); mientras que los geólogos parecían más razonables y accesibles. Como se puede observar aquí, el trauma del arqueólogo en ceder partes de una pieza arqueológica (con mayor razón si es "artística"), siempre ha existido.

Luego, se emprendió una campaña internacional para la obtención de muestras. Dentro de este contexto, es probableque el material requerido de Huaca Prieta llegara a Chicago para datación. De hecho, la primera muestra peruana en ser fechada es la C-75, un trozo de algarrobo de una vivienda Cupisnique y en asociación con maíz, a la época, por medio de solid carbon con un valor de $2665 \pm 200$ RYBP (i.e. $415-968 \mathrm{cal} \mathrm{AC} \mathrm{a}$ $68.2 \%$ de probabilidad, y con la curva SHcal04).

Durante la actividad de lahoratorio de Chicago, principalmente entre marzo de 1949 y principios de 1954, se fecharon más de 500 muestras. La primera lista de fechados fue publicada por mimeógrafo el 1 de enero de 1950 (Taylor 1987: 166). La misma, con algunas modificaciones, es la que aparece en febrero de 1951 (Arnold and Libby 1951), lo que incentivó a los laboratorios a presentar sus propias listas (Taylor 1987: 166).

Rápidamente el radiocarbono cobra auge, y es así que ya desde inicios de la década del 50 el solid carbon se implementa en 8 lahoratorios en el mundo, que incluyen la Universidad de Arizona, Universidad de Copenhague, Lamont Geological Observatory, Universidad de Columbia, Universidad de Michigan, Instituto de Estudios Nucleares de Nueva Zelandia, 
Universidad de Pennsylvania, Universidad de Saskatchewan, y Universidad de Yale (Taylor 1987: 167).

Pocos años después, algunos laboratorios iniciaron programas frente a la frecuente contamiración generada por el salid carbon. Es asíque los lahoratorios de Heidelberg en Alemania, Groningen en Holanda y el del US Geological Survey comenzaban a operar sus muestras por gas counting a partir de $\mathrm{CO}_{2}$ o acetileno (Taylor 1987: 168). Para la inauguración de la revista Radiocarbon ya habían unos 20 laboratorios activos y en implementación a nivel mundial, entre ellos el British Museum y Cambridge (Inglaterra), Colonia y Hannover (Alemania), Gif y Saclay (Francia), Stockholm y Uppsala (Suecia), Dublin (Irlanda), Lovaina (Bélgica), Moscú (Rusia), Roma y Pisa (Italia), Berna (Suiza) y Trondheim (Noruega) (Taylor 1987: 168).

El desarrollo inicial del método llevó a grandes contribuciones en el mismo Estados Unidos ya desde la década de 1950. En el Este, con fechados de maíz en el Alto Missisipi hacia el $2000 \mathrm{BP}$, generando así el debate sobre inicios de la agricultura. En el Oeste, la gran erupción del volcán Mazama, Oregon, fue fechada hacia el 6,800 BP. Ello posibilitó que este evento volcánico pudiera ser vinculado con ocupaciones humanas como teminus ante quem o teminus post quem, una suerte de marcador cronológico. Incluso el famoso Kennervick Man puede también datarse en función de su posición debajo del estrato de ceniza del volcán (Taylor 2000: 5-6).

Fue también desde esta década que Hans Suess, llegado de Europa con experiencia previa en radioactividad en la Universidad de Hamburgo, Alemania, empieza una campaña de colaboración con Libby. Luego trabaja con el profesor Fergusson, quien estaba dedicado a la recolección de muestras para fechado pordendrocronología en el lahoratorio de la Universidad de Arizona. El profesor Fergusson comprobó las variaciones de producción de $\mathrm{C}^{14}$ atmosférico que ya había hallado previamente el Dr. Vries (Suess 1992).
Producción de Radiocarbono

Dos principios básicos previos son la naruraleza química y la masa atómica de los elementos. El primero es la diferenciación entre elementos por presentar distintos números de protones en sus núcleos, lo que se conoce también como número atómico. El segundo es simplemente la suma del número de protones y neutrones en el núcleo de un átomo. Los átomos de un elemento tienen siempre la misma cantidad de protones en sus núcleos, no siendo así la cantidad de neutrones, que al variar, pueden cambiar la masa atómica. Justamente los átomos de esta variación de neutrones son conocidos como isótopos, aun manteniendo el mismo número atómico del elemento (Damon 1992; Taylor 1987: 1-5).

El carbono tiene también una naturaleza química característica. Forma parte de los seres vivos y es imprescindible en los procesos biológicos de los carbohidratos, proteínas, lípidos, etc. De manera natural, el carbono tiene 3 isótopos. Esto implica que los átomos de carbono tienen al 6 como número atómico. Sin embargo, por presentar diversos números de neutrones se producen tres isótopos de carbono $\mathrm{C}^{12}, \mathrm{C}^{13}$ y $\mathrm{C}^{14}$. Todos ellos suelen estar presentes en todos los compuestos de carbono.

No obstante, el $\mathrm{C}^{14}$ es el único de los tres isótopos que es radioactivo, i.e. su núcleo es inestable. Por esta inestabilidad, es posible que pueda transtormarse en $\mathrm{N}^{1+}$ emitiendo una partícula heta con carga eléctrica, lo que hace muy sensible su detección dentro de una muestra orgánica, aún cuando su presencia sea tan haja comn proporcionalmente la de un átomo $\mathrm{C}^{14}$ por mil millones de átomos de $\mathrm{C}^{\prime 2}$.

$\mathrm{El}$ isótopo $\mathrm{C}^{\text {li }}$ se está generando siempre en la biosfera. Se produce por el hombardeo constante de rayos cósmicos con núcleos de $\mathrm{N}^{14}$. Una vez está en la biostera, se combina con oxígeno para formardióxido de carhono $\left(\mathrm{CO}_{2}\right)$. Este $\mathrm{CO}_{2}$ se distribuye por toda la biosfera en océanos, lagos, ríos, y por medio de la fotosíntesis y la cadena alimenticia entre plantas y animales, incluyendo los seres humanos (Taylor 1987:2). 
A su muerte, un ser vivo deja de consumir $C^{\prime 4}$. Debido a su incstabilidad, su concentración empieza a disminuir según una proporción permanente. La vida media es una característica de átumos radioactivos. En el $\mathrm{C}^{1 ;}$ es de $5730 \pm 40$ años -Cambridge Half-Life, i.e. CHL. (Taylor 1987: 4). Previamente, Libby la había estimado en 5568 años. Sin embargo, avances en medición determinaron que ese valor estaba en ca. $3 \%$ menos que el real. Por ello, fechas obtenidas a partir del valor 5568 años, deben ser multiplicadas a $1.03 \%$, para su conversión en CHL. Ello es importante pues involucta, al menos, los fechados del laboratorio de Chicago, obtenidos en sitios clásicos como Huaca Prieta, Lauricocha, incluyendo algunos pioneros fechados en Huaca Santa Cruz (Mochica), Paracas e incluso Cahuachi, en el caso que tratamos ahora, para la cronología andina.

La norma pues se formulaba como: menos presencia de $\mathrm{C}^{14}$ en una muestra, más antigüedad. A partir de la existericia de la mitad de vida de un átomo $\mathrm{C}^{\mathrm{li}}$, el decaimiento (i.e. la transformación de $\mathrm{C}^{14}$ en $\mathrm{N}^{14}$ ) es más lento progresıvamente entre aproximadamente el 12000 y el 40 000 BP. Entre 40 y 50 mil años atrás hay muy poca cantidad de radiocarbono presente en la muestra. La unidad de dessintegración radiocarbónica por segundo se conoce como "Bequerel". La desintegración es constante, pero a la vez, espontánea, lo que hace necesario el uso de métodos estadísticos en el conteo (Lazos 2005).

La lista de organismos que pueden ser fechados viene incrementándose cada día de manera impresionante (cf. Hedges 1992: 175, Taylor 1987: 43-44). Ella incluye carbón, madera, celulosa de madera, madera fósil (la cual ha permitido calibración de alta resolución desde 14 milenios atrás), hojas de árboles, trigo, paja, humus, turba, plantas, granos (incluido maíz), grafito, colágeno y apatita de huesos ácido oxálico, frutas, plantas acuáticas, orģanismos marinos, hierbas, grass de zonas áridas, semillas, mijo, cuero, piel, cabello, pelos, y coprolitos, caparazones de huevo, $\mathrm{CO}$ : atmosférico, carbonatos naturales, suelos con $\mathrm{CO}_{2}$, agua marina, agua de ríos, lagos y lagunas, fango y sedimentos de lagos, bloques de hielo, polen, incluso cerámica, escoria metálica, piedra, pinturas murales, y de abrigos y cuevas, cuando hay existencia de carbono en sus componentes. También hierro, meteoritos, corales (que hace posible ahora la calibración hasta ca. 24000 años atrás), tufia, residuos sanguíneos, textiles y tejidus, papel, restos de peces, de insec. tos, resinas, $y^{\prime}$ cornamentas.

\section{Procedimiento para fechar muestras}

El procedimiento para fechar muestras se inicia desde el recojo de campo con la selección de la muestra, pre-tratamiento de ella para determinar el valor isotópico $\mathrm{C}^{13}$, y finalmente la conversión para la lectura de la muestra en años radiocarbónicos. Adicionalmente, se requiere calibrar el resultado obtenido, en función de convertirlo en años reales calendarios.

La primera fase del trabajo, i.e. la selección de la muestra, es extremadamente importante. El ideal es una selección conjunta de arqueólogo con la participación directa de al menos un representante del laboratorio al que va a ser remitida (Lazos 2005: 184).

De acuerdo a Hedges (1992: 165-183), existe una serie de parámetros recomendables para la selección de las muestras a fechar. Ellos se pueden resumir en dos. En primer lugar, cerciorarse de que la muestra está vinculada al evento a fechar. La pregunta es: ¿que relación tiene la muerte del organismo con el evento a fechar? Ello también implica un estudio detallado del contexto y del suelo donde está depositada la muestra. Este punto es fundamental, ya que no pocos casos demuestran errores de muestreo. Luego, habría que agregar observaciones de problemas tales como percolación, permafrost ${ }^{\prime}$, incluso, desplazamiento de material por causas rafonómicas.

En segundo lugar, se requiere de una evaluación para descartar posible contaminación de la 
muestra con otro tipo de madera (madera vieja). Por otro lado, fichas descriptivas del entorno de donde se ha recogido la muestra pueden ayudar mucho a los laboratoristas. Son también importantes las observaciones del arqueólogo de campo sobre los suelos de crecimientos de raíces, fango, proximidad a asfalto, productos derivados del petróleo y fósiles orgánicos como carbón natural (Taylor 1987: 40).

A pesar de los avances en cuanto al tamaño mínimo de muestra fechable por el AMS, es siempre recomendable manejar la muestra con pinzas esterilizadas $y$ depositarla en cajas de metal limpias o en su defecto, papel aluminio (vidrios pueden romperse). Nunca usar bolsas plásticas, de papel o de algodón (que no es raro ver en múltiples proyectos andinos). Cuando es imprescindible tocar el material con las manos, evitar que tengan grasa, manchadas de petróleo, carbón en polvo, o crema de manos (Taylor 1987: 68). A falta de cajas de metal hay también la costumbre (nuevamente en el área andina) de envolver cuidadosamente la muestra en papel aluminio, no obstante, la integridad de la muestra así no queda luera de posibles presiones, fraccionamientos y hasta trituramientus causados por material depositado de la excavación. Después de todo, justamente ya que el AMS trabaja con una fracción tan mínima, ello le hace más susceptible no solo a contaminación, sino al riesgo de la selección de una parte de la muestra no representativa (cf. Bronk Ramsey et al 2004). Por último, nunca rotular la muestra, sino solo por medio de una etiqueta sobre la parte externa del pomo o caja (Taylor 1987:68).

Muestras de carbón para conteo de rutina suelen pesar entre 2 y 40 gr (12 gr en carbón como óptimo peso), mientras que para AMS de 0.5 a 1 gr. Para bivalvos marinos se necesita usualmente entre 10 y 100 gr., y 10 g para AMS. En caso de plantas, granos, hojas y coprolitos entre 5 y 60 gr. para métodos convencionales y 1 a 2 gr. para AMS (Taylor 1987: 67).

El tratamiento de la muestra para la determinación de sus isótopos es la segunda fase del procedimiento. Esta etapa también es crucial pues es aquí donde sucede la purificación química y el aislamientode contaminantes. La selección de la parte más pura va desde sencillos procedimientos como la extracción de partes mezcladas con impurezas, microorganismos, raíces, etc., hasta el usode medios bioquímicos y lavados ultrasónicos (Taylor 1987:41,60). Cuidado especial merecen las muestras más antiguas debido a la exigua cantidad residual de $\mathrm{C}^{14}$ frente al $\mathrm{C}^{\prime 2}$. Por tanto, nos dice Lazos (2005), este tipo de material es más sensible a la contaminación. Ello sin embargo, no implica que materiales más recientes no puedan contaminarse, dependiendo de la historia individual de cada muestra.

Muestras orgánicas terrestres, tales como carbón o madera depositadas en un suelo, aun por poco tiempo, han absorbido generalmente, variadas proporciones de carbonatos inorgánicos, frecuentemente carbonato cálcico $\left(\mathrm{CaCO}_{\text {; }}\right)$ por medio de percolación de agua o humedad basal. Generalmente, el uso de ácido clorhídrico ( $\mathrm{HCl}$ ) remueve tales carbonatos absorbidos (Taylor 1987: 41). En otras circunstancias, si la muestra está impregnada por otros materiales orgánicos, ellos pueden ser removidos por solventes apropiados, como benceno, éter, metanol o tolueno, aunque los solventes también contienen carbón (Taylor 1987: 42). De igual manera, muestras almacenadas en laboratorios $y$ en museos por largo tiempo generalmente han sido manipuladas, lo que también resulta en contaminación. Por ello es sumamente importante incluir en el reporte si se ha usado algún preservativo (Taylor 1987: 42).

\footnotetext{
' Cubierta permanente de hielo sobre yacinientos arqueológicus, frecuentemente del Pleistoceno, y de latitudes altas.
} 
Desde el contador de radiocarbono hasta el AMS

El conteo Beta de radiocarbono directamente in carbón natural, se lliamó carbón sólido (so. lid carbon counting). En él se usaba un screen wuall counter y un contador Geiger. Se obtenía $\mathrm{CO}_{2}$ de la muestra por medio de la combustión o acidificación de ella. Luego, este gas era convertido por magnesio en carbón sólido (elemental), "carbón negro", cuyo muestreo era insertado directamente en el contador. Usualmente 12 gr. de carbón eran requeridos para un fechado con errores frecuentes de 200-300 años para muestras de hasta 5000 años (Taylor 1987: 82). Sin embargo, su precisión era del ca. $5 \%$ en el conteo del $\mathrm{C}^{14}$, a lo que había que sumar la frecuente contaminación por productos de fisión. Bluck carbon method fue dcjado de emplear alrededor de 1956, cuando Lihby dejó el Laboratorio de Chicago (Taylor 1987: 82).

Por los problcmas expuestos con el carbón sólido y avances en técnicas de conteo de gas procedentes de investigación nuclear, esta técnica fue reemplazada y superada rápidamente por conteo de gas (gas counting), donde la muestra es convertida en dióxido de carbono $\left(\mathrm{CO}_{2}\right)$, o un gas hidrocarbónico sintetizado de $\mathrm{CO}_{3}$, elevando la precisión a $95 \%-99 \%$ y demostrando una eficiencia operativa y más práctica (Taylor 1987: 83). Además, por enriquecimiento isotópico, las muestras podían ser fechadas ahora 75000 años, y sólo entre 1 y 5 gr. de carbón eran necesarios para edades como 40000 años. Incluso, instrumentos de alta precisión redujeron los errores a un sigma de hasta 15 y 20 años. Las muestras requcridas bajo este método generalmente producían entre $\pm 200-500$ años $C^{14}$ de precisión, y eran procesadas en promedio entre 48 y 120 horas (Taylor 1987: 96, table 4.1).

En la técnica llamada liquid scintillution (cintilación líquida), las muestras también son convertidas en $\mathrm{CO}_{2}$, para luego ser sintetizadas en benceno $\left(\mathrm{C}_{h} \mathrm{H}_{t}\right)$, el cual es inyectado por una solución cintilante que produce pulsos de luz en presencia de irradiación ionizada (Pollach 1992,
Taylor 1987: 76-77). Esta técnica fue desarrollada paralelamente al conteo de gas, y había sido usada en Chicago y Dublin. Sin embargo, fue ampliamente aceptada desde inicios de la década de 1960. Es desde esta época cuando se empezó a usar benceno por su alto contenido de carbón, además de poseer excelentes propiedades cintilantes. De hecho sigue siendo el método más frecuente en los laboratorios (Taylor 1987: 88-90).

Tanto para conteo por gas como también para liquicd scincillation, una muestra promedio puede oscilar entre 0.25 y $5 \mathrm{gr}$, cuyo resultado promedia $\pm 40-150$ y un procesamiento de entre 24 y 72 horas superando de lejos al solid carbon method. Sin embargo, aun bajo ambos métodos, para alta precisión se requieren entre 10 a $20 \mathrm{gr}$ de muestra, elevando la exactitud en un promedio de \pm 20-40 (Taylor 1987: 96, Table 4.1).

Los primeros ensayos con el AMS se dieron a fines de la década del 70. Ciertas imprecisiones, consecuencia del manejo químico por los anteriores métodos de conteo por gas, impulsaron la investigación para lograr sistemas de conteo directo (Taylor 1987: 90). Fue así como surge el AMS. La ventaja que proporcionaba era simplemente la disponibilidad de medir por conteo directo la masa isotópica del Carbono, que es la cambiante, según hemos visto bajo el principio de radioactividad (Hester 1987. Taylor 1987: 73). Previo pre-tratamiento, la muestra es ionizada por medio de la inserción de electrones en la cubierta externa de los átomos. Luego, los iones son canalizados en un tubo acelerador y polarizados por concentraciones debido a un campo magnético ejercido en el rubo. El grado de polarización de los iones depende mucho de la masa de cada uno de ellos. Luego, los iones seleccionados para el análisis alcanzan el colector de salida, quedando en el tubo por efecto de inercia y de excesiva polarización (Gove 1992). Finalmente, se les bombea fuera del espectrómetro y son leídos por medio de la energía de iones presentes, determinando así las concentraciones isotópi- 
cas. En mediciones de rutina, se contrasta la concentración de $C^{13}$ versus $C^{12}$ (Tavlor 1987: 73. 74). Una muestra peruana procedente de la cueva de Pikimachay tiene la virtud de ser la primera en el mundo en ser fechada por el Laboratorio de Berkeley AMS, en 1978, corrigiendo el valor de 5080 \pm 60 (UCLA-2118D) por el de 5900 \pm 800 (Taylor 1987: 94). Medidores de tercera generación, hacen posible determinaciones de rutina usando solo miligramos de muestra de carbón (Taylor 1987: 95). De hecho, AMS solo requiere de 2 a 5 miligramos, pudiendo ser medidos en 1 a 2 horas, y logrando una precisión de $\pm 80-400$ (Taylor 1987: 96, Table 4.1).

\section{Convenciones}

Para obtener la fecha de una muestra, por lo general se asumen ciertos criterios básicos (Stuiver and Polach 1977, Taylor 1987: 3):

- La concentración de $\mathrm{C}^{14}$ ha permanecido siempre constante,

- La existencia de mezcla rápida y completa a través de reservorios de carbón,

- Las proporciones de $\mathrm{C}^{14}$ no han sido alteradas,

- La vida media del átomo de $\mathrm{C}^{\text {Ht }}$ es precisa,

- Los niveles naturales de $\mathrm{C}^{14}$ son medidos a precisión.

BP

La noción de BP (Before Present) es simplemente la fecha que proporciona el laboratoriv en años radiocarbónicos antes de 1950 (año límite de los primeros fechados).

CRA

Otra convención extremadamente importante es que los laboratorios informan sus resultados en CRA (Conventional Radiocarbon Age), que significa que no es la edad real, la cual solo puede ser obtenida luego de la calibración. Algunos laboratorios presentan ya la calibración en sus informes, según la dendrocurva del momento, pero es imprescindible disponer siempre del CRA con el número correspondiente de laboratorio $y$, si es posible, el valor del fraccionamiento $\mathrm{C}^{13}$.
Contaminación y fraccionamiento isotópico

Una medición de $\mathrm{C}^{14}$ en una miuestra asume la pureza de carbón natural y su reducción a través del tiempo. Sin embargo, existe una serie de efectos físicos que pueden alterar los contenidos originales de $\mathrm{C}^{14}$. De hecho el problema más común en las mediciones de muestras es la contaminación por la introducción de carbón extra, alterando el contenido natural $C^{14}$ (Taylor 1987: 34-35).

El fraccionamiento incluye alteraciones de las proporciones isotópicas como la función de la masa atómica. $\mathrm{El} \mathrm{C}^{14}$ es mas "pesado" que el $\mathrm{C}^{13} \mathrm{y}$ que el $\mathrm{C}^{\prime 2}$. Empero, en procesos como la fotosíntesis isótopos más livianos son incorporados en la muestra. Por este motivo pueden suceder variaciones en las proporciones de $\mathrm{C}^{114}-\mathrm{C}^{12}$, que no guardan ninguna relación con la medición del tiempo (Taylor 1987: 35). Los valores de fraccionamiento dependen de cada tipc de material. Van desde $\mathrm{CO}^{3}$ marino, cuyo $\mathrm{C}^{13}$ es $\mathrm{O} \pm 2$, pasando por plantas como maíz con $10: \pm 2$, hasta carbón con $25 \pm 3$.

Sin embargo, las reacciones químicas no son las únicas que pueden generar fraccionamiento isotópico. La evaporación y la conclensación pueden también conducir a ello. El carbono disuelto en el mar, por ejemplo, se enriquerce en $0.7 \%$ en comparación con el $\mathrm{CO}_{2}$. Los laboratorios que disponen de AMS suelen presentar un informe del valor del fraccionamiento isotópico por mues. tra. Esta es presentada por la proporción entre $C^{13}$ y $C^{12}$, y se mide por un espectrómetro de masas. Se expresa por A 13 (i.e. edad normalizada) que significa las partes per mil entre el $C^{13}$ yel contenido internacional de carbonato (PDB), i.e. la desviación del PDB standard.

\section{Efecto Reservorio (Reservoir Effect)}

Es el cambio aparente originado por el depósito original de carbón con una anımalía de bajo $o$ alto contenido de $\mathrm{C}^{14}$. Si la mucstra se ha formado en un medio que tenga un contenido diferente al del $\mathrm{C}^{14}$ atmosférico, entonices el fechado radiocarbónico será mayor o menor que la edad 
dendro dependiendo de si contiene más o menos $\mathrm{C}^{\text {it }}$ que la atmósfera. Los bivalvos marinos cercanos a litorales tienen edacles radiocarhónicas de aproximadamente 350 años más que otros organismos terrestres porque el mar tiene un contenido menor de $\mathrm{C}^{\text {14 }}$ que la ar:músfera (Taylor 1987 : 33-34). Sin embargo, aparentemente, no sólo los medios ambientes son capaces de alterar el valor de $\mathrm{C}^{14}$ de una muestra. Mediciones $\mathrm{C}^{14}$ de muestras en distintas latitudes de la tierra de un mismo tiempo han resultado distintas. Como resultado del escudo magnético de la Tierra, los polos ejercen más actividad neutrónica, puesto que los rayos cósmicos son 5 veces más potentes que los del ecuador geornagnético. De ello se puede deducir que en el área andina, la actividad $\mathrm{C}^{1+}$ ha sido menor comparativamente hablando. Para hacer un poco más complejo el panorama, los estudios dendrocronológicos han demostrado que también existe un efecto reservorio entre árboles que crecen en zonas de más y menos altitud, con una variación de $\mathrm{C}^{14}$ en $\pm 0.5 \%$ (ca. 40 años) (Taylor 1987: 7). Y si bien puede tratarse de variaciones mínimas cuando tratamos de eventos arqueológicos como los andinos, no sabemos aun cuan "mínimas" puedan ser, en vista de la carencia de este tipo de estudios fundamentales en los Andes. Ello también incluye áreas de mayor radiación solar, pue:s tal como se ha comprobado hay una mayor actividad $\mathrm{C}^{14}$ en áreas insoladas.

\section{Efecto Suess, o Industrial.}

Desde mediados del siglo XIX, el uso cada vez más intensivo de combustibles fósiles $e$ industriales han incrementado dramáticamente el $\mathrm{CO}_{2}$ atmosférico. Como resultado de ello, la actividad radiocarbónica se ha reducido drásticamente, con una disolución de $2 \%$ en la primera mitad del siglo $X X$, haciendo poco recomendable el uso de madera que: ha desarrolladodesde 1890 (Suess 1992, Taylor 1987: 36-37).

\section{Efecto Carbono Bomba}

Otro factor que ha incrementado considera. blemente el $\mathrm{C}^{14}$ atmosférico es la serie de pruebas de armas nucleares y deronaciones entre $1945 y$ mediados de los años 70. El enorme incremento) del thujo de neutrones termales produjo artificialmente la casi duplicación del $\mathrm{C}^{14}$ en el Hemisferio Norte (Taylor 1987: 37-38) e incluso (algo que nos toca directamente), lo aumentó en un $60 \%$ en el Hemisferio Sur, cuyo pico máximo fue alcanzado en 1965. Investigaciones del proyecto WOCE (World Ocean Circulation Experiment) demuestran que el Pacífico es un área afectada por el Efecto Carbono Bomba, lo que permite sugerir la posibilidad de efectos colaterales en este lado del Pacífico Este (Nydal 2000), i.e. área cercana a los Andes.

\section{Desviación Standard}

Por razones estadísticas, se impone el promediar el rango de distribución del resultado. Ningún resultado puede ser infinito, lo que obliga a usar conceptos estadísticos para representar de manera exacta las distribuciones (Taylor 1987: 103). Por convención, los laboratorios presentan sus resultados con una desviación standard (1 sigma), lo que implica un 68\% de probabilidad (aproximadamente un tercio de 3). De igual forma, aproximadamente el $95 \%$ de probabilidad implica 2 sigmas, y finalmente $99 \%$ de probabilidad equivale a 3 sigmas. No obstante, el valor de 1 sigma es el mínimo valor en comparaciones de rutina de estimaciones de edades $\mathrm{C}_{\mathrm{H}}$ (Taylor 1987: 104). Ahora bien, los programas de calibración disponen de elección de uno, dos o tres sigmas, que deberán ser aplicados en función estricta del fenómeno a comparar. Secuencias con una alta cantidad de fechados pueden ser evaluadas por I sigma, mientras que secuencias más espaciadas y con menos valores $\mathrm{C}^{4 t}$, probablemente sean mejor evaluadas por 2 . o eventualmente 3 sigmas, dependiendo del nivel de probabilidad deseado. 
Evaluación de los fechados radiocarbónicos

Uno de los grandes aportes del radiocarbono es su función para estimar edades, sobre todo en sociedades ágrafas. De hecho, importantes observaciones tales como establecer cronologías absolutas para la Prehistoria de la humanidad en el Pleistoceno Terminal, pueden ser posibles gracias al radiocarbono. La misma importancia no sucede para épocas de las cuales se guarda registro escrito (Taylor 1987: 106). Precisamente este es el caso de los Andes, puesto que es bien sabida la falta de registro escrito hasta antes de la Conquista. Ello ha hecho a la cronología absoluta de la arqueología andina casi dependiente del radiocarbono. Precisamente, este es uno de los motivos por los cuales, los arqueólogos que trabajan con este tipo de "prehistoria" andina, deberían convertir los fechados radiocarbónicos en calendarios para poder saber con certitudlos verdaderos tiempos en que sucedieron los hechos.

En cuanto a fechados erróneos, ya sean demasiado recientes o demasiado antiguos, vale la pena reproducir la tabla que Taylor nos ofrece (Ver Tabla 1).

Uno de los factores más importantes a evaluar, son el tipo de carbón a extraer como muestra. En especial, el carbón de procedencia aluvial, e incluso de permafrost deben ser tratados con cautela, pues son conocidos los problemas de desviaciones radiocarbónicas (Taylor 1987: 109-110). En la evaluación de fechados radiocarbónicos también hay que prestar atención a perturbaciones generadas, por e.g. bioturbación (frecuentemente causada por micromamíferos) o por factores antropogénicos. Sitios como cuevas y abrigos son afectados por este tipo de alteraciones (Taylor 1987: 111).

También hay que tener muy en cuenta el efecto reservorio. El caso tipico es fechar organismos con contenido $\mathrm{C}^{14}$, menor en variados porcentajes en comparación con los otros organismos vivientes. Los bivalvos marinos, o conchas, son frecuentemente sujeto de este problema (Hedges 1992: 171). Sin embargo, puede tam. bién afectar a peces que se alimentan de sedimento lacustre. Los valores referenciales de efectos reservorio para muestras marinas para, al menos la costa norte, la central y la sur del Perú, pueden ser consultados en Marine DateBase de Paula Reimer (Reimer y Reimer 2001, http://www.qub.ac.uk/arcpal/marine). Tales valores son de fundamental importancia cuando se calibran fechados sobre este tipo de materiales, frara lo cual hay una serie de dispositivos provistos en cada programa de calibración.

Otro valor reservorio interesante es el que se deriva de fechados en moluscos terrestres. El carbonato de la concha de un caracol terrestre, por ejemplo, puede contener una importante cantidad de carbonatos, diferente a su medio ambiente (є..g. Hedges 1992: 172). El área andina no escapa a este tipo de problemas, tal como el caso de algunos caracoles terrestres fechados para el ComplejoPaiján (cf. Chauchat etal 1992).

Dentro de lo valores reservorio terrestres hay que tener en cuenta a las plantas que estuvieron expuestas a vientos de fumarolas o de erupciones volcánicas, cuyos valores $\mathrm{CO}_{2}$ no contienen $\mathrm{C}^{\mathrm{H}}$ por su propia naturaleza geológica (Hedges 1992: 171). Este $\mathrm{CO}_{2}$ magmático al mezclarse con el $\mathrm{CO}_{2}$ atmosférico reduce la actividad radiocarbónica en áreas generalmente pequeñas. Las áreas del conocido Eje Neovolcánico del occidentede Mexico, donde la historia de la actividad volcánica ha sido y aún es intensa, son sujeto de: tales contenidos alterados $\mathrm{de}^{\mathrm{C}^{14}}$ atmosférico $y$, por tanto, de fechados absolutos. Investigaciones llevadas a cabo en el Mediterráneo y Europa sobre actividad volcánica prehistórica han resultado en variaciones entre 1400 y 4000 años de error (Taylor 1987 131-132). Una aproximación similar sería interesante para el área central andina, en función de documentar áreas de actividad volcánica en relación con posibles alteraciones de $\mathrm{C}^{14}$ atmos. férico. Arequipa puede ser un buen sujeto de estudio en este sentido, para empezar, si se tiene en consideración la alta actividad volcánica e 
Principales causas de fechados radiocarbónicos anómalos

\begin{tabular}{|c|c|}
\hline \multirow{12}{*}{$\begin{array}{l}\text { Edad } \\
\text { aparentemente } \\
\text { más reciente que } \\
\text { lo esperado }\end{array}$} & $\begin{array}{l}\text { - Errónea identificación de la muestra con respecto a su verdadera } \\
\text { procedencia estratigráfica y conitexto. }\end{array}$ \\
\hline & - Depósito original de la muestra, en situación de mezcla debido a \\
\hline & bioturbación y geoturbación. \\
\hline & - Remoción insuficiente de raíces de carbón y hueso. \\
\hline & - Remoción insuficiente de productos húmicos de degradación orgánica, \\
\hline & percolados desde niveles estratigráficos superiores. \\
\hline & - Aplicación inadecuada de valores de corrección reservorio. \\
\hline & - Descuido del depósito de las muestras o depósitos inadecuados de \\
\hline & muestras (bolsas de papel o cartón, bolsas de tela). \\
\hline & - Materiales preservantes usados en las muestras, producidos por fuentes de \\
\hline & carbón modernas. \\
\hline & - Muestras rotuladas erróneamente. \\
\hline \multirow{11}{*}{$\begin{array}{l}\text { Edad } \\
\text { aparentemente } \\
\text { más antigua que } \\
\text { lo esperado }\end{array}$} & - Errónea identificación de la muestra con respecto a su verdadera \\
\hline & procedencia estratigráfica y contexto. \\
\hline & - Deriósito original de la muestra, en situación de mezcla debido a \\
\hline & bioturbación ygeoturbación. \\
\hline & - Remoción insuficiente de productos húmicos de degradación orgánica \\
\hline & procedentes de depósitos más antiguos. \\
\hline & - Mezcla con fuente de carbón fósil (asfalto, lignita, alquitrán). \\
\hline & - Remoción insuficiente de carbonatos de agua de lagos y lagunas. \\
\hline & - Aplicación inadecuada de valores de corrección reservorio. \\
\hline & - Materiales preservantes usados en las muestras, producidos por fuentes de \\
\hline & carbón modernas. \\
\hline
\end{tabular}

Tabla 1. Principales fuentes de valores $C^{\text {it }}$ para contextos arqueológicos típicos (tomado de Taylor 1987: 107).

incluso las capas de ceniza volcánica producidas por la erupción del volcán Huaynaputina, registrada para el $1600 \mathrm{AD}$, y sus potenciales consecuencias en el contenido $\mathrm{C}^{14}$ de depósitos arqueológicos anexos.

Calibración radiocarbónica y las nuevas curvas IntCal04 y SHCal04

El impacto de la calibración radiocarbónica fue tal que hasta Renfrew se atrevió a tildarla como la "segunda revolución radiocarbónica", por sus grandes aportes en la cronologia absoluta de Europa, desde las tumbas megalíticas hasta los tempranos templos de Malta, que como consecuencia, fueron sujeto de revisiones importantes (1973). Luego de las calibraciones radiocarbónicas, varios planteamientos difusionistas quedaron atrás, estableciendo, por ejemplo, que las tumbas megalíticas eran más antiguas que las pirámides egipcias, los templos de Malta sorprendentemente más antiguos que los del Cercano Oriente, e incluso, que la metalurgia cuprifera de los Balcanes era más antigua que en la propia Grecia (Renfrew 1973).

Por otro lado, la calibración radiocarbónica se hizo poco a poco más necesaria, no sólo por la 
necesidad de correlacionar eventos en el pasado fechados por otros métodos (TL, OSL, Hidratación de Obsidiana, erc.) que proporcionan resultados calendarios, sino también cuando se los trata de insertar en el medio ambiente, cl cual también se suele plantear en años calendarios. Por tanto, al parecer, la historia del área andina, estrictamente desde este punto de vista de años calendarios, está aún por escribirse.

De hecho, el esfuerzo en cuanto la conversión de años radiocarbónicos en calendarios para una mejor aproximación y evaluación de eventos pasados data casi desde el descubrimiento del método del radiocarbono. Se partió entonces de la premisa de que la intensidad constante de rayos cósmicos había sido errada. Es más, no se habían tenido en cuenta los cfectos de ella sobre el campo geomagnético terrestre, y otros efectos de la actividad solar sobre la del $\mathrm{C}^{\prime 4}$ atmosférico (Becker 1992, Stemberg 1992). Para solo citar un ejemplo de efecto geomagnético, la variación es tan alta que desde hace $20 \mathrm{kyr}$ en $400 \%$ se redujo hasta $100 \%$ en el 10,000 BP (cf. Sternberg 1992: 99). En cuanto a la relación entre actividad solar y posibles cambios climáticos, se ha planteado la relación entre oscilaciones climáticas y reducciones de temperatura a veces drásticas- en directa relación a un aumento de $\mathrm{Al} 4 \mathrm{y}$, a su vez, baja actividad solar. De ellos el periodo Spoerer entre ca. 1380-1520 es uno de los más fuertes, que si bien está satisfactoriamente documentado para el Hemisferio Norte (Damon y Jirikowic 1992), suscita preguntas como algún posible impacto en el área andina, en el contexto del final del Periodo Intermedio Tardío y el inicio del Horizonte Tardío. Mas aún, sobre la base de la quasi simultaneidad de eventos medioambientales entre el Hemisferio Norte y el Sur (Rothlisberger 1986).

La historia de los inicios de la dendrocronología data de 1906 hasta los años 30, cuando A. E. Douglass hace los primeros ensayos en USA (Douglass 1935, citado por Becker 1992: 34), sobre todo en función de reconstrucciones de clima en el pasado. Uno de los descubrimientos más. importantes de Douglass fue que los anillos de coníferas presentaban un crecimiento reducido durante épocas secas. Poco tiempo después, un colaborador de Douglass, Schulman, descubrić árboles en California que le posibilitaron presentar una secuencia dendro de 4600 años (Becker 1992: 35). Inspirado en este logro, Bruno Huber, un botánico alemán, a base esfuerzos de correlacionar secuencias dendrocronológicas europeas, logra fechar varias culturas del Neolítico (Huber y Merz 1963, citado por Becker 1992: 38).

Hacia fines de los años 50, pruebas coordinadas de medición de los laboratorios de Carnbridge, Copenhagen, y Heilderberg produjeron una serie de determinaciones del $\mathrm{C}^{\text {Ht }}$ sobre muestras de anillos de árboles, demostrando una variación consistente en el $\mathrm{C}^{\text {li }}$ biosférico en alto poricentaje durante los últimos 1300 años (Willis et al 1960). Este hallazgo no hacía mas que confirmar el pionero descubrimiento de De Vries en 1958, cuando se refirió a que los años radiocarhónicos no dehen ser asumidos como equivalentes a años siderales o calendarios (De Vries 1958). De hecho, hoy en día, se conoce al Efecto De Vries como sinónimo de las oscilaciones de la curva (wiggles) (Taylor 1987: 30).

Desde aquellos tiempos, han pasado ya más de cuatro décadas de esfuerzo de un buen núme. ro de laboratorios tratando de documentar las diferencias sistemáticas entre los años radiocarbónicos y los años calendarios (Taylor 1987: 133).

En esta historia, hay dos hitos importantes. En primer lugar, el esfuerzo en la creación de una curva a base de múltiples series-dendro (Arizona, Groningen, La Jolla, Pennsylvania y Yale) que: alcanzaba hasta 7200 años. En segundo lugar, la recomendación dada en el $12^{\circ}$ International Radiocarbon Conference (1985) sobre el uso oficial de las curvas de calibración de Stuiver y Pearson para el periodo entre el 2500 AC' hasta el presente (Taylor 1987: 133), para 
posteriormente ampliarla hasta el 5000 AC (Stuiver and Pearson 1992). A partir de esta fecha se han generado nuevas curvas en búsqueda te alta precisión de calibración, además de una generación de al menos 6 softuares, tanto europeos como norteamericanos, para microcomputadores sencillos, de modo tal, que cada investigador puede contar con esta valiosa herramienta en su PC. La curva, además, se ha ampliado por medio del uso de madera fósil, y sobre todo rebasa el Lust Glacial Muximum ${ }^{2}$ a base de mediciones $\mathrm{U} / \mathrm{T}^{3}$ en corales (cf. Bard y Menot-Combes 2004, Becker 1992: 47, Kitagawa y van der Plicht 2000, Stein et al 2000, Stuiver y Pearson 1992: 32). Por último, la aplicación de la estadística bayesiana en función de complementar la precisión de la calibración, i.e. de usar el teorema de Bayes en función de convertirlo en una herramienta matemática para invertir la calibración en probabilidades, ha hecho de ella una herramienta bastante precisa (Buck et al 1996), a pesar de recientes críticas (Steier y Rom 2000).

Una rápida exploración de la última curva de calibración es suficiente para observar anomalías que pueden repercutir en el planteamiento de las cronologías absolutas clásicas del área andina. Es evidente que en muchos ejemplos, como las secciones de la curva de calibración, un mismo fechado radiocarbónico puede tener múltiples equivalentes fechados calendarios (i.e. plateaux). De acuerdo a Taylor, esto implica que para algunos periodos, el tiempo radiocarbónico es "elástico", i.e. puede ser "alargado" o "acortado". Un "plateau" famoso es el llamado "Hallstart" (en homenaje a la cultura suiža del primer milenio AC). De esta forma, fechados de entre 2400 y $2530 \mathrm{BP}$, se pueden distribuir en un plano casi horizontal que se encuentra aproximadamente entre el 410 y 790 AC, i.e. ca. 380 años de imprecisión. Dentro de este mismo periodo, uno puede poner en duda la validez de gran parte de la secuencia radiocarbónica del Horizonte Temprano andino, incluyendo la posición calendárica verdadera de fases dentro del Horizonte Temprano (en especial Urabarrio). Una investigación relativamente reciente muestra que este periodo está determinado por una alteración de rayos cósmicos y por la trayectoria crrática virtual del polo geomagnético terrestre durante ca. 2800-2200 BP. A este fenómeno se le conoce como "Stemo-Etrussia" Geomagnetic Excursion (Dergachev et al 2004).

Ahora bien, iqué necesitamos para evaluar y calibrar un fechado radiocarbónico? Dos fuentcs. En primer lugar, obviamente el informe correspondiente del contexto de donde procede la muestra. Hay que recordar que se pueden obtener una serie de fechados en laboratorios le prestigio internacionales con última tecnología. Sin embargo, si la muestra procede de un contexto disturbado, no se relaciona con el evento a fechar, o si incluso hay sospechas de contaminación, el fechado no tiene validez. De modo que la importancia del contexto se haya en directa proporción al mismo procedimiento del fechado radiocarbónico en el laboratorio. En segundo lugar, disponer, en la medida de lo posible, de! informe del propio laboratorio para evaluar el pretratamiento mecánico y químico, y el propio fechado.

Cuando se tiene el informe del laboratorio en la mano, hay que asumir convenciones como la edad media de decaimiento (o transformación radiocarbónica), ya sea la de Libby (5568), o Cambridge (5570), el uso de 1950 como O BP, el uso directo o indirecto de NSB ácido oxálico usado frecuentemente, la normalización de las muestras a base de su fraccionamiento isotópico y la aceptación de la constancia de niveles de $\mathrm{C}^{\text {H4 }}$ en la atmósfera durante el tiempo (Taylor 1987: 137). Estos datos deben estar en el informe, así

\footnotetext{
2 Último Avance Glacial entre el 18000 BP y el Dryas Ill (ca. 11000 BP).

Método de fechado por Utanum/Thorium.
} 
como también, si son disponibles, valores reservorio (en caso de muestras carbonatadas), que en todo caso dependen estrictamente de la localidad de donde la muestra procede, y deberían ser discutidos con el director del laboratorio.

En cuanto a las desviaciones standard, ellas son necesarias, por los motivos ya expuestos. Laboratorios convencionales suelen presentar el fechado con un sigma de error (68\%). Doblar este error nos aproxima al $95 \%$ de probabilidad, i.e. dos sigmas. Siguiendo el manual de Taylor, podemos ofrecer fechados absolutos a modo de ejemplo, pero esta vez usando algunos de los Andes y con la curva SHCal04 (2005), en función de evaluar la diferencia de uno y dos sigmas.

En el primer caso, en la muestra ISGS-507: $2400 \pm 100$ RYBP, para Chakinani, se observa el típico problema ya identificado como "DeVries wiggles", i.e. los llamados "plateaux". Bajo ambas probabilidades las variaciones son tan grandes, que poco sirve todo ensayo de precisión, pues ambas fechas se distribuyen sobre 4 o 5 centurias calendáricas de imprecisión, de modo tal que este fechado simplemente es un indicio de la distorsión de gran parte de la cronología absoluta para Chavín de Huántar. Un test de calibración sobre todas las muestras procedentes del sitio, y bajo contexto bien controlado y documentado puede ser revelador, no sólo para este sitio clave para la cronología andina, sino también para el Horizonte Temprano.
El segundo caso es tomado del fechado Gd6196: $810 \pm 70$ RYBP sobre madera, aparentemente hallada en una tumba Chancay. El resultado es $1190 \mathrm{cal}$ AD- $1296 \mathrm{cal}$ AD con un sigma de probabilidad. El valor relativamente bajo de la desviación standard, así como la situación de la muestra sobre la curva donde hay poca variabilidad o anomalías de producción $\mathrm{C}^{14}$, le confieren una aproximación bastante aceptable y compatible con la cronología tradicional de la cultura Chancay.

\section{Problemas con el radiocarbono en los Andes}

Pensamos que poco hay que agregar a las acertadas observaciones que se han vertido previamente por el proyecto de catalogación de fechados radiocarbónicos andinos (Ziólkowski et al 1994). Doce años han pasado ya de esta interesante publicación que pretendió la ambiciosa tarea de reunir todos los fechados radiocarbónicos andinos. Sin embargo, por el contenido de este trabajo, vale la pena recordar los 7 puntos planteados. No vamos a abundar en detalles. El lector puede consultar directamente el trabajo original (Ziólkowski etal 1994:9-21).

El primer problema concierne a los valores consignados como vida media del radiocarbono (Ziólkowski et al 1994: 9). De hecho, sí bien la variación es mínima, $y$ viene ya en el paquete del programa moderno de calibración, es necesario tenerla en cuenta en la lectura e interpretación del fechado radiocarbónico.

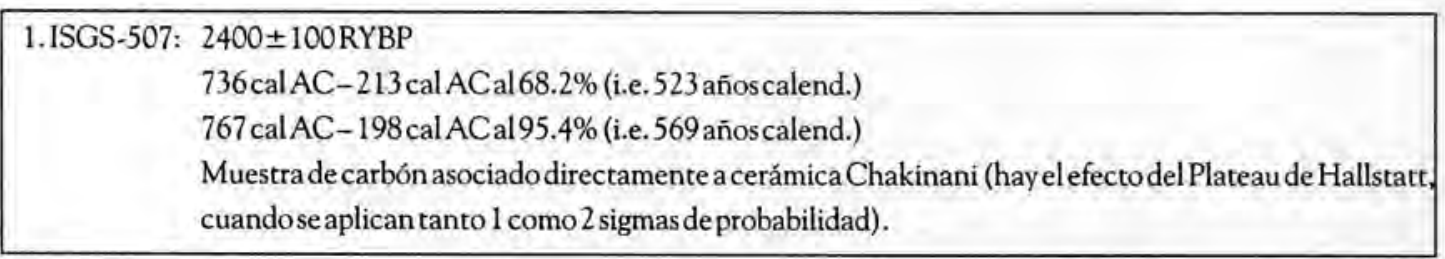

2.Gd-6196: $810 \pm 70$ RYBP

$1190 \mathrm{cal} A D-1296 \mathrm{cal} A D$ al $68.2 \%$ (i.e. 106 años calend.)

1052 cal AD- 1389 cal ADal $95.4 \%$ (i.e. 337 añoscalend.)

Muestra de madera, supuestamente de contexto funerario asociada a cerámica Chancay (la muestra también se ve afectada por un pequeño plateaux entreaproximadamente 1000 y $1200 \mathrm{AD}$ ). 
Figura 1. Parte $\mathrm{AD}$ de la Curva de Calibración SHCal04 (MacCormac et al 2004). Nótese la irregularidad de los conocidos wiggles (cf. De Vries 1958) y cómo pueden afectar parcialmente la cronología de varias de las secuencias prehispánicas peruanas -e.g. Moche, Lima, Horizonte Mediosólo en esta parte de la curva.

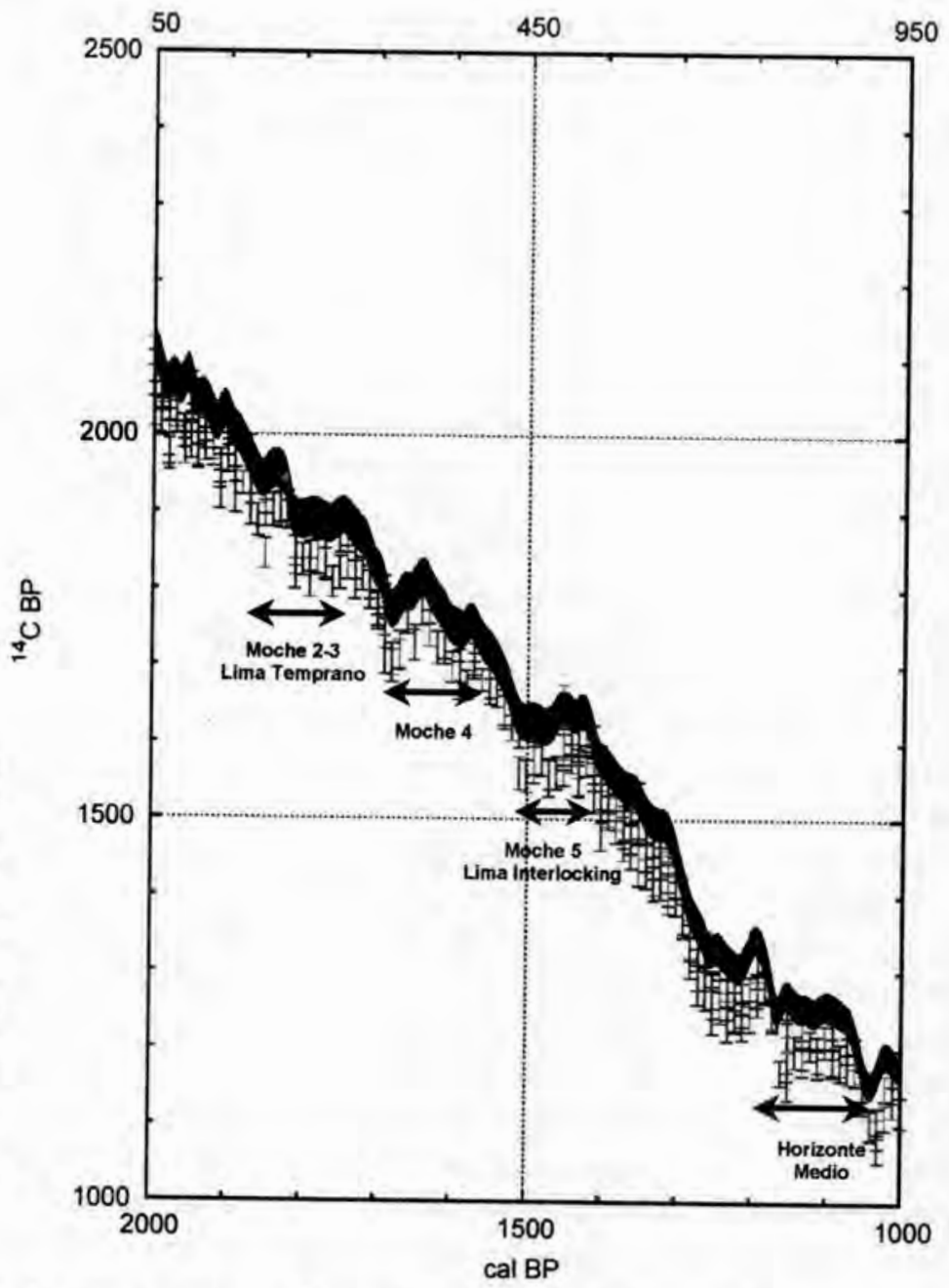

Un segundo punto es el de la precisión del método usado. Bien nos recuerda Ziólkowski que varios de los fechados andinos son tan antiguos como el nacimiento del mismo método de radiocarbono. Por consiguiente, una serie de fechados han sido obtenidos por medio de solid carbon, un método, como hemos visto, con un potencial grande de contaminación pretratamiento (ut supra). El resultado de la comparación de fechados de la misma muestra por varios laboratorios demuestra claramente la imprecisión de este método, al menos hasta su funcionamiento, a mediados de los años 50 . Tal es el caso de la misma muestra fechada por Lamont
Observatory y el Laboratorio de Chicago con un rango de margen error de más de 600 años radiocarbónicos (cf. Ziólkowskietal 1994: 10).

El tercer problema, i.e. el "Suess Effect", resulta ahora casi mínimo en vista de las correcciones automáticas de los programas de calibración informáticos. Empero nos hacen tomar ciertas precauciones cuando se trata con muestras fechadas por solid carbon, hasta antes de la introducción del ácido oxálico NBS como standard.

Un cuarto punto toca al fraccionamiento isotópico. La práctica de la mayoría de los laboratorios hoy en día de proporcionar fechados "normalizados" (normalized), es relativamente 
reciente. Es por ello, que muchas muestras de las primeras décadas de fechados deben ser tomadas con un error por no haber efectuado fraccionamiento isotópico y detectar el conteniulo especifico $\mathrm{C}^{\text {t4 }}$ de ellas. Mas cuidado aun merece el caso de fechados en organismos marinus con alto contenido de carbonatos y en organismos terrestres como el maíz.

El quinto problema expuesto puede parecer absurdo, sin embargo es le consideración. Se trata de los frecuentes errores cuando se citan los fechados en diferentes publicaciones. En principio, se entiende que todos los fechados deben ser siempre expresados en BP (AP) o AD (DC), sin embargo, es evidente que por descuidos e igno. rancia se los han reportado de las más diversas formas (cf. Ziólkowski 1994: 12-13). Por consiguiente, hay que prestar siempre atención a la publicación original, o probar por respuesta directamente de la fuente, i.e. el laboratorio.

Los laboratorios brindan actualmente el fechado radiocarbónico y la calibración con la última curva en uso. Usualmente se coloca 1 sigma como rango de probabilidad. Sin embargo, uno puede aplicar dos y hasta tres sigmas de probabilidad, de acuerdo al sottware. Se impone también la selección de la curva IntCal04 (para el Hemisferio Norte) o Shcal04 (para el Sur). En este procedimiento, nosotros no estamos de acuerdo con Ziólkowski cuando él con su equipo no estiman importante ni adecuado insertar en la calibración el Error del Hemisferio Sur. A doce años de la imprecisión impuesta por la única referencia del Hemisferio Sur (Pretoria, Sudáfrica), hoy en día hay una serie de puntos de referencia donde se han obtenido valores en función de establecer no sólo errores de esta índole (MacCormac et al 2002, 2004), sino incluso de disponer de toda una curva de calibración para el Hemisferio Sur, a la cual nos acabamos de referir, y que promete grandes cambios en la cronología absoluta del área andina (Fig. 1). Nosotros la recomendamos para los fechados andinos, pues a pesar de que los andes centrales están cerca del
Ecuador, la Shcal04 es más apropiada para nuestra área de investigación versus la lntCal04. La única limitación es que la dendrocurva Shcal04 termina antes, en 9682 años racliocarbónicos antes del presente, y por tanto no cubre por ahora el Pleistoceno Terminal.

La estimación de los errores por desviación standard debe ser también tomada en cuenta, pues en algunos casos, al parecer no hay referencias ni en la literatura, ni por medio del laboratorio. Pueden haber también errores de los propios laboratorios, falta de código por el frropio laboratorio, errores en cadena por f́lta de mantenimiento o control del laboratorio, hasta falta de información sobre localización y contexto de las muestras.

Nosotros agregaríamos, finalmınte, la necesidad de valores reservorio para el Perú, en función de contar con valores específicos de producción de $\mathrm{C}^{\text {t }}$ en los Andes.

\section{Efectos de la calibración radiocarbónica en la arqueología andina}

Pocos, pero notorios y prometedores, son los recientes trabajos de calibración con material andino en función de evaluar secuencias (Adamska 1991, Lau 2004), incluso en contrastación con fechados TL que sugieren radicales modificaciones cronológicas para Moche (Roque et al 2002). Sin pretender semejante análisis, a continuación vamos a hacer un solo ejercicio de calibración y evaluar la importancia de su aplicación en términos de precisión cronológica. La invitación para usar la curva y" explorar sus efectos en la arqueología andina está dada.

\section{El Complejo Ayacucho}

En vista de la potencial importancia del Complejo Ayacucho, aún a pesar de estar sustentado por una sola fecha, se le ha tomado como ejemplo. Hay que enfatizar que una tase debe estar sustentada por al menos una serie de fechados radiocarbónicos para sumar probabilidades. No se ha considerado al Complejo Pacaicasa por 
las críticas ya expuestas (Rick 1988: 13-16). Solamente hay una muestra que fecha este Complejo, lo que de ningt:na manera es suficiente para considerar a ésta como segura. Lo único que se puede concluir, es que es un indicio de la posible ocupación más antigua de los Andes centrales hasta el momento. Vamos ahora a tratar de la muestra, su resultado y su procedencia con asociaciones.

La muestra UCLA-1 464 dio un valor medio de 14150 180 (MacNeish et al 1981: 209). Aunque no estamos en contra del "promedio", mas aún tratándose de uria sola muestra, pensamos que sería interesante incluir el conteo completo de la muestra para la calibración, tratándose probablemente de un fechado tan relevante. La medición se hizo por colágeno, en 1969, por medio del método de gas counting, resultando específicamente en tres lecturas: 14180 a los 2800 minutos, 14150 a losi 1300 minutos y 14080 a los 1200 minutos (MacNeish et al 1981: 209, 212). Esta nisma muestrix se cita en la compilación de fechados radiocar|bónicos del grupo polaco, empero erróneamente, como I-1464 y sin la calibración respectiva, pues en esa época aún la curva de calibración no había sido configurada para el Pleistoceno Final (cf. Ziólkowski et al 1994: 323). De igual moilo, hay (que añadir que no hay un reporte especifico del pretratamiento de la pieza de hueso, en vista de las críticas vertidas al fechado de hueso (e.g. Tisnerat-Laborde et al 2003).

La muestra fue una porción de húmero de perezoso (probablemente: Scelidoterium) encontrada en la unidad S19.1E3, dentro del estrato "h" de la Cueva de Pikimachay (Ac100), a una profundidad de $2.67 \mathrm{~m}$. La capa " $h$ ", de acuerdo al plano de la figura. 2.39 (MacNeish et al 1981: 49), se extiende al menos por $103.8 \mathrm{~m}^{2}$, entre las unidades $\mathrm{S} 11$ y $\mathrm{S} 25$, y E1 y E14. Los autores presentan 5 cortes del área excavada. Sin embargo, lamentablemente, ninguno sobre el área del propio contexto (cf. MacNei:sh et al 1981: 44-47), lo que hubiera graficado mejor la situación estrati- gráfica. Lo único que se puede concluir de los planos presentados es que, en efecto, la capa es bastante homogénea. En ningún caso a modo de lente, lo que sugiere un argumento a favor desde el punto de vista estratigráfico

Resulta curioso que en el informe final de las exc:avaciones no haya un gráfico que permita evaluar el contexto (MacNeish et al 1981). Esta documentación clave para examinar en detalle tal ocupación está publicada en un artículo (MacNeish 1979). Sobre la capa "h" entonces, se pueden apreciar cinco concentraciones llamadas áreas deactividad $1,2,3,4$ y 5 . La muestra procede del área de actividad $3, y$ a pesar de que no está expresamente señalizada, ha podido ser ubicada exactamente sobre el punto referido S19.1E3 con el código Z1-11 (cf. MacNeish 1979: 35, fig. 19). El fragmento procede entonces, del área centro-este de dicha concentración. En general, el área de concentración 3 es de contorno irregular, de aproximadamente $3.57 \mathrm{~m}$. de largo por $3.71 \mathrm{~m}$. de ancho, con una profundidad entre 7 y $13 \mathrm{~cm}$, por tanto relativamente reducida, lo que recuerda típicas concentraciones del Paleolítico Superior europeo (e.g. Cziesla 1990). El tipo de huesos sugiere que se trata del mismo animal.

El fragmento de húmero datado se encuentra rodeado de otros huesos, también generalmente de la misma especie. Un primer punto a considerar es qué fecha es el hueso que se ha datado. De allí la importancia del contexto. Hay dos posibilidades: 1) Que el hueso proceda de un evento animal natural o incluso tafonómicu, e.g. bioturbación; o 2) Que haya sido transportado antrópicamente. Dado los especímenes hallados en el contexto del área de actividad 3, i.e. artefactos líticos, su distribución en quasi forma de abanico, desechos de talla, posiblesáreas de combustión cercanas e incluso huesos con huellas de corte -aún cuando falte documentación apropiada, e.g. Haynes 2000- es posible plantear la hipótesis de que el hueso de húmero fechado proceda de un evento producido por humanos. 
Es importante tambiérı señalar la presencia de algunos artefactos que resultan claves en el propio contexto, aunque por la corta distancia podría considerarse todo como de un mismo evento. Examinemos primero a los más cercanos. En un radio de un me:tro hay que destacar un fragmento de costilla de Scelideterium con huellas de corte, y sobre todo una serie de piezas dentales, probablemente del rnismo animal. En un radio de 2 a 3 metros hallamos al menos 12 supuestos utensilios líticos agrupados a modo de abanico con orientación Este-Sur-Oeste en el sentido de agujas de reloj. Se trata de un hendidor unifacial en guijarro (B7), tres raederas en guijarro (SS55), tres lascas con borde utilizado (SS6), un supuesto buril (\$S58), dos denticulados grandes (SS52 y SS53i), una raedera sobre laja (SS50), y una raedera sobre lasca (SS21). También hubieron tres extremos poximales de lascas (P) (MacNeish 1979: 35, fig. 19). Se ha tratado de ubicar la descripción o material gráfico de esta colección y no sie ha tenido éxito. Sin embargo, al menos dos fotografías dan cuenta del material existente en las demás áreas de concentración que en todo se hallan en un radio de 6.5 $\mathrm{m}$ desde la muestra fechada, i.e incluyendo las 5 áreas de concentración. Si bien la fotografía no permite observar con detalle la posible tecnologia lítica de esta serie, nos permitimos sugerir la posible veracidad de retoque sobre piezas que podrían clasificarse comodenticulados y posiblemente un hendidor unifacial (cf. MacNeish 1979: $44-45$, figs. $22.6,22.7$ y 23.3, 23.14). Es también significativo que este material esté tallado sobre materia prima de alta silicificación a juzgar por el lustre que se puede observar en contraste de negativos, y más aun por la organización de ellos.

Sobre esta misma capal también se ha publicado una punta de proyectil de hueso (MacNeish 1979: 35, fig. 19, concentración de la izquierda). Si bien, lamentablemente se hizo una foto del espécimen, éste permite observar la posibilidad de pulimento sobre ambos bordes, lo que habría resultado en una silueta bastante homo- génea (MacNeish 1979: 45, fig. 23.4, MacNeish et al 1980: 309, fig. 8-1, espécimen superior derecho). Es de forma triangular y está elaborada en un fragmento de un metapodial de un caballo fosilizado (MacNeish et al 1980: 309). La punta mide $46 \mathrm{~mm}$ de largo por $25 \mathrm{~mm}$ de ancho. Dos de las principales características de esta pieza es la presencia de pulido en varios sectores, incluyendo reducción basal, y una serie de líneas y marcas mayormente verticales, sobre todo en el extremo distal (MacNeish et al 1980: 309 y 312 ).

Otra observación interesante es la presencia casi exclusiva de huesos largos en el área de concentración 1 de Scelidoteriım, lo que para MacNeish responde a caza y descuartizamiento de los animales antes de ser transportados a la cueva (MacNeish 1979: 37).

En cuanto a las áreas de combustión también de la misma área, los autores mencionan el hallazgo de tres rocas posiblemente termofracturadas en ordenamiento circular, en un área de suelo enrojecido. Además, cuatro huesos oscurecidos por posible evento de combustión han sido reportados (MacNeish et al 1980: 7). Este material procede del área de concentración 5 (MacNeish 1979: 38-39). Esta evidencia es extremidamente importante en vista de la posibilidad de eventos de combustión de carácter antropogénico. De hecho el fragmento de húmero fechado no dista más de 6 metros hacia el oeste de este material combustionado (cf. MacNeish 1979: 34-35, figs. 18 vs. 19), lo que se podría considerar una evidencia más de ocupación humana, ahora más bien deste un punto de vista espacial, siempre en referencia al hueso fechaclo.

El estrato " $h$ ", de $10 \mathrm{~cm}$ de espesor en promedio, aumenta hasta $30 \mathrm{~cm}$ hacia la boca de la cueva (MacNeish 1979: 33). Aquí se hallaron mas cle 100 "artefictos" líticos, 42 huesos de animal modificados antrópicamente con marcas de corre, pulidos y marcas aserradas (MacNeish 1980: 6), restos vegetales como Chcnopodiaceue (MacNeish et al 1975: 14-15) y de semillas de Almus, los que sugieren que tal tipo de alimentación fue accesible (MacNeish et al 1980: 6). 
En suma, a pesar de la poca documentación específica y la errónea identificación de material lítico (tal como en el caso de los "buriles"), en el balance se puede afirmar que algunos artefactos líticos aparentemente de material silificado, huellas de uso en huesos, concentración de artefactos, selectividad de huesos importados en la cueva y las posibles áreas de combustión expuestas podrían indicar que estamos en presencia de la ocupación más antigua de los Andes.
Veamos ahora el resultado de la calibración. Tendríamos en la figura 2 un fechado corregido a 15 271-14 610 años calendarios $\mathrm{AC}$, con un sigma de probabilidad. Hay que señalar que puede existir una variación en esta precisión, pues la Shcal04 no cubre este periodo, por lo que este fechado ha sido calibrado con la IntCal04 para el Hemisferio Norte. No obstante, creemos que la diferencia no debe ser grandemente significativa. Es interesante comprobar que por efec-

CALIBRADIOCARBONCALIBRATIONPROGRAM*

Copyright 1986-2005MStuiver andPJ Reimer

*Tobe used in conjunction with:

Stuiver, M., and Reimer, PJ., 1993, Radiocarbon, 35, 215-230.

UCLA-1464

BoneScelidoterium-humerus-

Radiocarbon Age $14150 \pm 180$

Calibrationdata set: intcal04.14c

\# Reimeretal. 2004

One Sigma Ranges: [start:end] relative area

[15271BC:14610BC] 1 .

TwoSigma Ranges: [start:end] relative area [15633BC:14329BC] 1 .

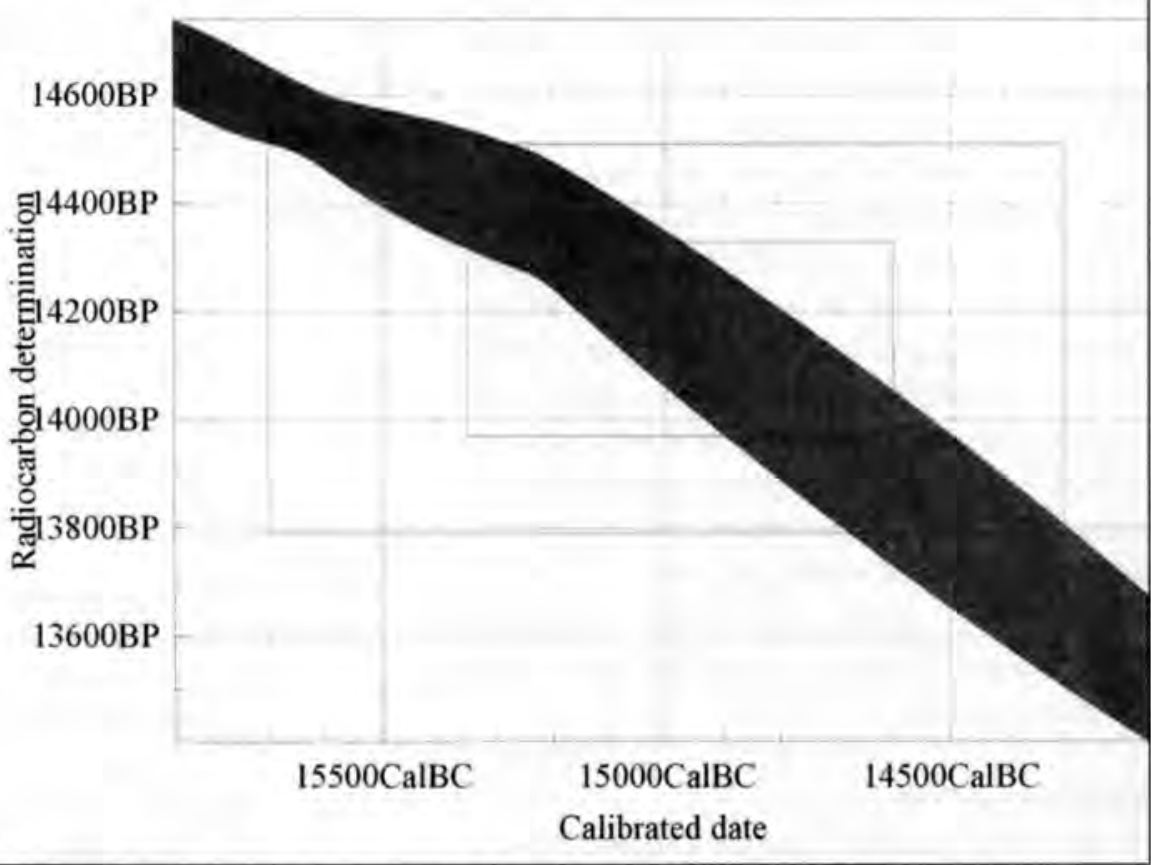

Figura 2. UCLA-1464 calibrado por medio de la IntCal04 a 1 sigma: 15271-14610 BC. 
to de posición en la curva el fechado resulta más antiguo de lo supuesto.

\section{Comentarios Finales}

Una de las mejores formas de compenetrarse con el radiocarbono y la calibración es mediante el conocimiento de su historia y repercusiones no sólo a nivel de investig'ación personal y local, sino también regional, y inás aun internacional. En ella, como hemos visto, Bird y Fejos han jugado un rol importante comos proveedores de mues. tras andinas peruanas, irıcluso desde el mismo nacimiento del método.

Manejar los principios del método del radiocarbono no es simplemerite el establecer fechados y corregirlos. Ellos son solo medios o herramientas en función de ubicar en el tiempo determinados eventos para luego interrelacionarlos, unos a otress, sin embargo, siempre descle la perspectiva necesaria de fechados corregidos, o calibrados, i.e. calendarios.

De igual forma, el contexto de donde procede la muestra es de extrenta importancia antes de rècoger la muestra o bien en el momento de su evaluación. No olvidemus la lección de Taylor, en la cual el arqueólogo es al final el responsable de la muestra, puestoque él la suministra.

El manejo del software de calibración, ya sea de los europeos o de los norteamericanos, no es difícil, pues solo $\mathrm{OxCal}$ dispone de un vasto manual que se haya en versión zip en el propio software. El día que se conciba la real importancia de la calibración radiocarbónica dentro del medio universitario peruano, se habrá avanzado un paso iınportante hacia la respuesta de la pregunta ¿cuándo pasó eso? Hace 2500 100 años radiocarbónicos o realmente entre 750 y 470 AC. Por tanto, significa el Horizonte Temprano desde el punto de vista radiocarbónico, i.e. cronología absoluta?

\section{Agradecimientos}

A Duccio Bonavia, por sus valiosos comentarios y correcciones. De igual mudo a Paula
Reimer (Belfast Laboratory, Irlanda) en cuanto al radiocarbono y calibración. Finalmente a Olaf Joeris (Laboratorio de Radiocarbono, Universidad de Colonia, Alemania) por haberme motivado en este campo durante mi doctorado en la Universidad de Bonn, A lemania.

\section{Bibliografía}

Adamska A.

1991 The Inca State Chronology in Light of Radiocarbon Dates. Master's thesis Department of History, Warsaw University.

Arnold, J. R. y W. F. Libby

1949 "Age Determinations by Radiocarbon Contene: Checks with Samples of Known Age". Science 1 10:678-680.

Arnold, J. R. and W. E. Libby

1951 "Radiocarhon Dates". Science 113: 111. 120.

Aurenche, O, P. Galet, E. Régagnon-Caroline, J. Évin

2001 "The Birth of Agriculture, Livestock Raising, and Ceramics: A Calibrated 14C Chronology 12,500-5,500 cal BC". Radiocarbon 43 (3): 1191-1202.

Bard, E., F. Rostek, y G. Menut-Combes

2004 "A Better Radiocarbon Clock". Science 303: 178-179.

Becker, B.

1992 "The History of Dendrochronology and Raaliocarbon Calibration". En R.E. Taylur, A. Long, and R.S. Kra (eds.). Radiocarbon After Four Decades. An Interdisciplinary Perspective, pp. 34-49. Springer Verlag. New York.

Braiwood, R. J., T. Jacohsen, R. Parker, and P. Weinberg

1951 "Radiocarbon Dates and their Implications in the Near and Middle Eastern Area. A Brief". En Radincarbon Dating, assembled by F. Johnson, pp. 54. 55. Memoirs of the Society for American Archaeology, N. 8. The Society for American Archaeology'. Salt Lake City. 
Bronk Ramsey, C., T. Higham, and P. Leach 2004 "Towards High-Precision AMS: Progress and Limitations". Radiocarbon 46 (1): 17-24.

Buck C. E., W. G. Cavanagh, and C. D. Litton

1996 Bayesian approach to interpreting archaeological data. Chichester, New York, Brisbane, Toronto, Tokyo, Singapore: John Wilcy \& Sons.

Chauchat, C. (with the collaboration ofE. S. Wing; J.-P. Lacombc; P.-Y. Dermars, S. Uceda Castillo \& Carlos Deza)

1992 Préluistoire de la Côte Nord du Pérou. Le Paijanien de Cupisnique. Cahiers du Quatcrnaire $N^{\circ}$ 18. Centre National de la Recherche Scientifique. Centre Régional de Publication de Bordeaux. CNRSÉditions. Paris.

Collier, D.

1952 "Radiocarbon Dating". American Anthropologist 54 (4):558-559.

Currie, L. A.

2004 "The Remarkable Metrological History" of Radiocarbon Dating". Journal of Research of the Nutional Institute of Standards and Technology 109: 185-217.

Cziesla, E. (ed.)

1990 The Big Puzzle: International Symposium on Refitring Stone Artefacts, Monrepus. 1987. Holos Verlag. Bonn.

Damon, P. E.

1992 "The Natural Carbon Cycle". En R.E. Taylor, A. Long: and R.S. Kra (eds.). Radiocarbon After Four Decades. An Interdisciplinary Perspective. pp. 17-18. Springer Verlag. New York.

Damon, P. E. and J.L. Jirikorvic

1992 "Solar Forcing of Global Climate Change?" En R.IE. Taylor, A. Long, and R.S. Kra (eds.). Radincarbon After Four Decades. An Interdisciplinar' Pcrspective, pp. 117-129. Springer Verlag. New York.

Daniel, G.

1967 The Origin and Growth of Archueology. Crowell.New York.
Dergachev, V. A., O.M. Raspupuv, B. Van Geel, G.

I. Zaitseva

2004 "The 'Sterno-Etrussia' Geomagnetic Excursion around 2700 BP and Changes of Solar Activity, Cosmic Ray Intensity, and Climate". Radiocarbon 46 (2): $661-681$.

De Vries, $H$.

1958 "Variations in concentration of radiocarbon with time and location on earth". Proceedings, Nederlandische Akademie van Wetenschappen, Series B 61: 1 .

Gove, H.E.

1992 "The History of AMS, its Advantages over decay counting: Applications and Prospects". En R.E. Taylor, A. Long, and R.S. Kra (eds.). Radiocarbon After Four Decules. An Interdisciplinary Perspective, pp. 214-229. Springer Verlag. New York.

Haynes, G.

2000 "Mammoths, Measured Tines, and Identities". Radiocarbon 42 (2): 257269.

Hedges, R.E.M.

1992 "Sample Treatment Strategies in Radiocarhon Dating". En R.E. Taylor, A. Long, and R.S. Kra (eds.). Radiocarbon Afere Four Decades. An Interdisciplinany Perspective, pp. 165-183. Springer Verlag. New York.

Heizer, R. F.

1956 "Radiccarbon Dating". American Anchropologist 58 (2): 394-395.

Hester, J.J.

1987 "The Significance of Accelerator Dating in Archaeological Method and Theory". Journal of Field Archaeology 14 (4): 445-451.

Johnson, $\mathrm{F}$.

1965 "The Impact of Radiocarbon upon Archaeology". En Chatters, R.M., Olson, E.A. (Eds.). Proceedings of the Sixch International Conjerence Radiocarbon and Tritium Dating, pp. 762. 780, Springfield, Virginia. 
Kitagawa, $\mathrm{H}$ and J. Van der Plicht

2000 "Atmospheric Radiocarbon Calibration Beyond 11,900 Cal Bp From Lake Suigetsu Laminated Sediments". Radiocarbon 42 (3): 369-380.

Lau, G.

2004 "The Recuay Culture of Peru's NorthCentral Highlands: A Reappraisal of Chronology and its Implications". Joumalof Field Archaeology 29: 177-202.

Lazos Ramirez, L.

2005 "Fechamiento por Radiocarbono". En: Rodrigo Esparza López y Efraín Cárdenas García. (eds.). Arqueometría. Técnicas Nucleares y Convencionales Aplicadas a la Investigación Arqueológica, pp: 181-196. El Colegiode Michoacán.

Libby, W. F.

1946 "Atmospheric Helium three and radiocarbon from cosrnic radiation". Physical Review 69: 671-672.

Libby, W. F.

1952 Radiocarbon Dating. University of Chicago Press. Chicago.

MacCormac, F. G., P.]. Reimer, A. G. Hogg, T. F. G. Higham, M.G. L. Baillie, J. F'almer, y R. Stuiver 2002 "Calibration of the Radiocarbon Time Scale for the Southern Hemisphere: A.D. 1850-950". Radiocarbon 44 (3): 641.651 .

MacCormac, F. G., A. G. Hogg, P. G. Blackwell, C. E. Buck, T. F. G. Higham, y P. J. Reimer

2004 "ShCal04 Southern Hemisphere Calibration, 0-11.0 Cal Kyr BP". Radiocarbon 46 (3): 1087-1092.

MacNeish, R. S., T. C. Patterson and D. L. Browman

1975 The Central Peruvian Prehistory Interaction Sphere. Papers of the Robert S. Peabody Foundation for Archaeology; vol. 7. Phillips Academy, Andover. Massachusetts.

MacNeish, R.S.

1979 "The Early Man Remains from Pikimachay Cave, Ayacucho Basin, Highland Peru". En Robert L. Humprey
\& Dennis Stanford (eds.). Pre-Llano Cultures of the Antericas: Paradoxes and Possibilities, pp 1-47. Anthropological Society of Washington.

MacNeish, R. S., R. K. Vicrra; A. Nelken-Turner, and C. J. Phagan

1980 Prehistory of the Ayacucho Basin, Peru. Volume III, Nonceramic Artifacts. Robert S. Peabody Foundation for Archaology. Ann Arbor. The University of Michigan Press. Ann Arbor.

MacNeish, R. S., A. G. Cook, L. G. Lumbreras, R. K. Vierra, and A. Nelken-Terner.

1981 Prehistory Of The Ayacucho Basin, Peru. Volume li, Excavations And Chronology. Robert S. Peabody Foudation For Archaology. Ann Arbor. The University OfMichigan Press. Ann Arbor.

Mellars, $P$.

2006 "A New Radiocarbon Revolution and the Dispersal of Modern Humans in Eurasia". Nature 439: 931 -935.

Nydal, R.

2000 "Radiocarbon in the Ocean". Radiocarbon 42 (1): 81-98.

Pollach, H.A.

1992 "Four Decades of Progress in 14C Dating by Liquid Scintillation Counting and Spectrometry". En R.E. Taylor, A. Long, and R.S. Kra (eds.). Radiocarbon After Fuur Decades. An Interdisciplinary Perspective, pp. 198-213. Springer Verlag. New York.

Reimer, P. J., Y R. W. Reimer

2001 "A Marine Reservoir Correction Database and On-Line Interface". Radiocarbon 43 (2A): 461-463.

Reimer, P. J., M. G. L. Baillie, E. Bard, A. Baylliss, J. W. Beck, C. J. H. Bertrand, P. G. Blackwell, C. E. Buck, G. S. Burr, K. B. Cutler, P. E. Damon, R. L Edwards, R. G. Fairbanks, M. Friedrich, T. P. Guilderson, A. G. Hogg, K. A. Huguen, B. Kromer, G. MacCormac, S. Manning, C. B. Ramsey, R. W. Reimer, S. Remmele, J. R. Southon, M. Stuiver, S. Talamo, F. W. Taylor, J. van der Plicht, C. E. Wehenmeyer 
2004 "IntCal04 Terrestrial Radiocarbon Age Calibration, 0-26 Cal Kyr BP". Radiocarbon 46 (3): 1029-1058.

Renfrew, C.

1973 Before Civilizatisn, the Radiocarbon Revolution and Prehistoric Europe. Alfred A. Knopf. New York.

Rick, J. IV.

1988 "The Character and Context of Highland Preceramic Society". En Richard W. Keatinge (ed.). Peruvian Archaeology. An Overview of Pre-Inca Society, pl: 3-40. Cambridge University Press. London.

Roque, C., E. Vartanian, P. Guibert, M. Schvoerer, D. Lévine, IV. Alva, y $H$. Jungner

2002 "Recherche chronologique sur la culrure mochica du Pérou : datation de la tombe du Prètre de Sipán par thermoluminescence $(T L)$ et par radiocarbone". Jounal de la Société des Américanistes 88: 227.243.

Rothlisherger, $M$.

1986 10,000 Jahre Ciletschersgeschichte der Erde. Verlag Sauerlander.

Savage, S.

2001 "Towards an AMS Radiocarbon Chronology of Predinastic Egyptian Ceramics". Radiocarbon 43 (3): 1255 1277.

Steier, P.y W. Rom.

2000 "The Use of Bayesian Statistics for 14C Dates of Chronolngically' Ordered Samples. A Critical Analysis". Radincarbon 42 (2): 183-198.

Stein, M., S. Goldenterg, A. Schramm.

2000 "Radiocarton Calibration Beyond the Dendrachronology Range". Radincarbun 42 (3): 415-422.

Stermberg, R. S.

1992 "Radiocarbon Fluctuations and Geomagnetic Field". En R.E. Taylor, A. Long, and R.S. Kral (eds.). Rudiocarbon After Four Decaules. An lnterdisciplinury
Perspective, pp. 93-109. Springer Verlag. New York.

Stuiver, M. y H. E. Polach

1977 "Discussion: Reporting of 14C Data". Radiocarbon 19: 355.363.

Stuiver, M. and G. W. Pearson

1992 "Calibration of the Radiocarbon Time Scale, 2500-5000 BC". En R.E. Taỵlor,

A. Long, and R.S. Kra (eds.). Radiocarbon After Four Decades. An Interdisciplinary Perspective, pp. 19-33. Springer Verlag. New York.

Suess, H. E.

1992 "The Early Radiocarbon Years: Personal Reflections". En R.E. Taylor, A. Long, and R.S. Kra (eds.). Radiocarbon After Four Decades. An Interdisciplinary Perspective, pp. 11-16. Springer Verlag. New York.

Taylor, R.E.

1987 Radiocarbon Dating. An Archaeolngical Perspective. Academic Press. New York.

Taylor, R. E.

2000 "The Contribution of Radiocarbon

Dating to New World Archaeology".

Radiocarbon 42(1). 1-21.

Tisnerar-Lahorde, N., H. Valladas, E Kaltnecker, y M. Arnold

2003 "AiMS Radiocarhon Dating Bones at LSCE". Radiocarbon 45 (3): 409-419.

Willis, E.H., H. Tauber, y' K. O. Munnich.

1960 "Variations in the Atmospheric Radiocarbon Concentration over the Past 1300 years". Radiocarbun 2: 1-4.

Ziólkowski, M. S., M. F. Pazdur, A. Krzanowski, and A. Michczyñski

1994 Andes. Radiocarbon Database for Bolivia, Ecuador, and Peru. Andean Archaeological Mission of the Institute of Archaeology, Warsaw University \& Gliwice Radiocarhon Laboratory of the Institute of Physics, Silesian Technical University: Warsawa Gliwice. 